\title{
T-wave Alternans Presence in Young Competitive Athletes - To Be or Not To Be Accepted as a Prognostic Factor?
}

\author{
Iana Simova $^{1}$, Ivan Gruev ${ }^{2,3}$, GIovanni Bortolan $^{4}$, Ivaylo Christov $^{5}$, Sofia Georgieva ${ }^{6}$ \\ ${ }^{1}$ Acibadem City Clinic Cardiovascular Center - University Hospital, Sofia, Bulgaria \\ ${ }^{2}$ Clinic of Cardiology, National Transport Hospital, Sofia, Bulgaria \\ ${ }^{3}$ Euro-Vita Sport and Dental Clinic, Sofia, Bulgaria \\ ${ }^{4}$ Institute of Neuroscience IN-CNR, Padova, Italy; \\ ${ }^{5}$ Institute of Biophysics and Biomedical Engineering, BAS, Sofia, Bulgaria \\ ${ }^{6}$ National Cardiology Hospital, Sofia, Bulgaria
}

\begin{abstract}
Objective We analysed the prevalence of TWA during cardiopulmonary stress test (CPT) in athletes and in patients with suspected or proven coronary artery disease with stress electrocardiographic test.

Materials and Methods Athlete's group comprised 414 subjects (24 \pm 8 years, $95.5 \%$ males), non-athlete's - 107 $(63 \pm 10$ years, $42 \%$ males). Stress testing lasted longer and heart rate $(H R)$ increased more in athletes compared to non-athletes. A TWA cluster was defined in case of uninterrupted appearance of TWA > 30 seconds.

Results Our initial analysis revealed higher prevalence and worse parameters of TWA in athletes compared to non-athletes: at least one TWA cluster in 389 athletes $(94.0 \%)$ and in $56(52.3 \%)$ non-athletes, $p<0.001$.

When we considered TWA-HR relationship and limited our TWA analysis to HR-range common to the two groups (80-125 bpm), our results changed significantly, with lower TWA prevalence in athletes than in non-athletes: 50 (12.1\%) vs 46 (42.9\%), $p<0.001$.

Conclusion TWA is a HR-dependent parameter and athletes achieving high HR during CPT have an increased prevalence of TWA. Therefore, the prognostic significance of exercise-induced TWA in athletes could not be the same as that of TWA in high-risk non-athletes, where TWA appears in a lower HR range.
\end{abstract}

\section{Introduction}

T-wave alternans (TWA) reflects instability of cardiac repolarization [1]. It is continuous variable with higher values implementing greater risk for sudden cardiovascular death (SCD) $[\underline{2}, \underline{3}]$. The current recommendation is that it is reasonable to consider TWA evaluation whenever there is suspicion of vulnerability to lethal cardiac arrhythmias [4]. Our research group has already performed several studies on this topic in various clinical settings: patients undergoing coronary artery bypass grafting [ $\underline{5}, \underline{6}]$, such with renal disease []], diabetes mellitus [8], during diagnostic stress ECG tests [9], during diagnostic test for Brugada syndrome [10].

On the other hand, elite athletes, despite their astonishing performance and absence of symptoms, are not immune from SCD and tailored screening programs have been developed to detect the presence of cardiovascular disorders and prevent SCD [11].

There is a shortage in the scientific literature of TWA studies in athletes. In a research on 85 athletes, all with ventricular arrhythmias, but without structural heart disease, TWA has been proven as a useful risk stratification factor [12].

Our aim was to analyse the prevalence of TWA during cardiopulmonary stress test (CPT) in athletes, using as a comparator a group with high expected TWA prevalence elderly patients with multiple risk factors, with suspected or proven coronary artery disease, performing diagnostic stress electrocardiographic test.

\section{Materials and methods}

\subsection{Study groups}

Cardiopulmonary stress test of young competitive athletes. The cardiopulmonary stress test data was collected at Euro-Vita Sport and Dental Clinic, Sofia, Bulgaria, as part of the annual work-up of competitive athletes. The database of the current study comprises 414 young competitive athletes, from the following sports: football, rhythmic gymnastics, badminton and tennis.

Diagnostic stress ECG test of non-athletes. ECG data of 107 non-athletes individuals was collected at the National Cardiology Hospital, Sofia, Bulgaria. Indication for stress ECG test in these occasions was diagnostic workup for patients with suspected myocardial ischemia. The stress test was performed using veloergometer (GE Marquette Stress PC ECG Application) - 2-min stages 
$25 \mathrm{~W}$ incremental workload.

\subsection{Signal preprocessing}

Signal preprocessing was performed on all recordings to eliminate/suppress the typical noise that accompanies the ECG. The $50 \mathrm{~Hz}$ interference was eliminated by the removal procedure of Levkov et al. [13], the electromyographic noise was suppressed by dynamic application of an approximation procedure [14] and baseline drift was suppressed by a high-pass recursive filter with a cut-off frequency of $0.64 \mathrm{~Hz}$ [15].

TWA was detected by analysis of: T-wave amplitude in a combined lead and T-wave complexity obtained by the method of Principal Component Analysis (PCA). The method was described in Bortolan and Christov, and approved in PhysioNet / Computers in Cardiology Challenge [16, 17].

We performed the TWA identification in the entire ECG recordings, and considered the presence of "TWA cluster" in case of a continuous occurrence of TWA for a minimum duration of 30 seconds.

\subsection{Ethics}

To be included in the registry, patients had to sign an informed consent for personal data analysis, and to agree to be followed up according to protocol. The study protocol is in accordance with the Declaration of Helsinki.

\subsection{Statistical analysis}

The distribution of continuous variables was tested using the Kolmogorov-Smirnov test. Normally distributed data were presented as mean \pm standard deviation, whereas non-normally distributed data - as median and interquartile range (the difference between the 25th and 75th percentile). Categorical variables were presented in percentage terms. We compared demographic characteristics and ECG features at rest and during exercise using Student's T-test for independent groups for normally distributed data and Mann-Whitney $U$ test for nonnormally distributed data.

\section{Results}

Our two groups were very different regarding demographic features, clinical characteristics and stress test parameters. Non-athletes, as compared to athletes, were older $(63 \pm 10$ vs $24 \pm 8$ years, $p<0.001)$, with more equal gender distribution ( $42 \%$ vs $95.5 \%$ males, $\mathrm{p}<0.001$ ). Certain clinical conditions were present only in the nonathletes group: arterial hypertension (90\%), dyslipidemy $(81 \%)$, obesity $(30 \%)$, diabetes $(36 \%)$, angina pectoris $(80 \%)$, history of myocardial infarction (17\%), angiographically significant coronary artery disease $(45 \%)$, history of stroke $(6 \%)$. In the latter group 34 patients $(32 \%)$ showed evidence of inducible myocardial ischemia during the stress test. The test was considered positive in the setting of $\geq 1 \mathrm{~mm}$ horizontal or downwardsloping ST depression $80 \mathrm{msec}$ after J-point.

\subsection{TWA analysis}

Stress testing lasted 15.3 (14.1-16.3) and 7.1 (5.5-8.4) minutes in the athletes and non-athletes groups, respectively, $\mathrm{p}<0.001$ and heart rate (HR) during stress was 145.6 (133.3-156.4) and 95.0 (86.7-105.0), respectively, $\mathrm{p}<0.001$.

Our initial analysis revealed higher prevalence and worse parameters (mean and max TWA amplitude) of TWA clusters in athletes compared to non-athletes: at least one TWA cluster in 389 athletes (94.0\%) and in 56 (52.3\%) non-athletes, $\mathrm{p}<0.001-$ Table 1.

Table 1 . TWA characteristics in athletes and non-athletes $(*: \mathrm{p}<0.01)$

\begin{tabular}{lccc}
\cline { 2 - 3 } & $\begin{array}{c}\text { Athletes } \\
(\mathrm{n}=414)\end{array}$ & $\begin{array}{c}\text { Non-athletes } \\
(\mathrm{n}=107)\end{array}$ & $\mathrm{p}$ \\
\hline 3 TWA clusters & $81(19.6 \%)$ & $1(0.9 \%)$ & $*$ \\
2 TWA clusters & $198(47.8 \%)$ & $17(15.9 \%)$ & $*$ \\
1 TWA cluster & $110(26.6 \%)$ & $38(35.5 \%)$ & - \\
no TWA clusters & $25(6.0 \%)$ & $51(47.7 \%)$ & $*$ \\
TWA duration & $3.97 \mathrm{~min}$ & $0.77 \mathrm{~min}$ & $*$ \\
& {$[2.3-6.1]$} & {$[0.53-1.47]$} & \\
TWA duration $(\%)$ & 26.0 & 11.6 & $*$ \\
& {$[15.6-39.7]$} & {$[7.6-19.1]$} & \\
TWA mean ampl. & $0.40 \mu \mathrm{V}$ & $0.36 \mu \mathrm{V}$ & - \\
& {$[0.22-0.84]$} & {$[0.16-0.68]$} & \\
TWA max ampl. & $0.84 \mu \mathrm{V}$ & $0.52 \mu \mathrm{V}$ & $*$ \\
& {$[0.44-1.77]$} & {$[0.31-1.04]$} & \\
Mean HR & $146 \mathrm{bpm}$ & $95 \mathrm{bpm}$ & $*$ \\
& {$[133-156]$} & {$[87-105]$} & \\
HR in 1 ${ }^{\text {st }}$ packet & $160 \mathrm{bpm}$ & $103 \mathrm{bpm}$ & $*$ \\
& {$[138-173]$} & {$[93-115]$} & \\
HR in 2 ${ }^{\text {nd }}$ packet & $146 \mathrm{bpm}$ & $92 \mathrm{bpm}$ & $*$ \\
& {$[125-170]$} & {$[79-109]$} &
\end{tabular}

\subsection{TWA-HR relationship}

Since TWA is a HR-dependent parameter, we considered the TWA-HR relationship. HR increase during testing was greater and faster in athletes compared to nonathletes and, consequently, HR distribution of the number of TWA clusters differed between groups (figure 1).

We considered the entire range of HR variation during stress test (50-230 bpm) and divided it in 12 intervals, each comprising a range of $15 \mathrm{bpm}$. We analyzed the presence of TWA clusters in each interval in both groups - Table 2 . The $2^{\text {nd }}$ and $4^{\text {th }}$ columns report the number of individuals 
achieving the corresponding HR during the test in the athletes' and non-athletes' groups, respectively. The $3^{\text {rd }}$ and $5^{\text {th }}$ columns report the corresponding number of individuals with a TWA clusters in the same range.
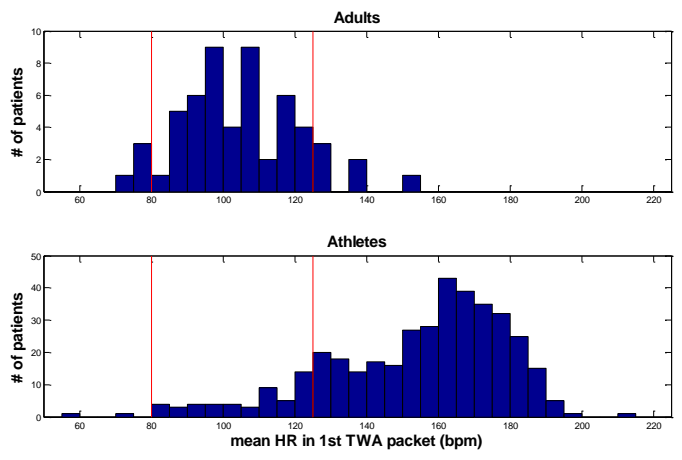

Figure 1. HR distribution of the number of TWA clusters in athletes and non-athletes; red lines delineating the region of interest.

Table 2. Presence of TWA clusters in the non-athletes' and athletes' groups, at different HR ranges

\begin{tabular}{rcccc}
\hline & \multicolumn{3}{c}{ Number of individuals } \\
\cline { 2 - 5 } HR & \multicolumn{2}{c}{ Non-athletes } & \multicolumn{3}{c}{ Athletes } \\
\cline { 2 - 5 } range & With a & With a \\
bpm & $\begin{array}{c}\text { Total n } \\
(\%)\end{array}$ & $\begin{array}{c}\text { TWA } \\
\text { cluster } \\
\text { n (\%) }\end{array}$ & $\begin{array}{c}\text { Total n } \\
(\%)\end{array}$ & $\begin{array}{c}\text { TWA } \\
\text { cluster n } \\
(\%)\end{array}$ \\
\hline $50-65$ & $5(4.7 \%)$ & $0(0 \%)$ & $29(7 \%)$ & $1(3 \%)$ \\
$65-80$ & $67(63 \%)$ & $4(6 \%)$ & $132(32 \%)$ & $1(1 \%)$ \\
$80-95$ & $100(93 \%)$ & $\mathbf{1 2 ( 1 2 \% )}$ & $295(71 \%)$ & $\mathbf{1 1 ( 4 \% )}$ \\
$95-110$ & $89(83 \%)$ & $\mathbf{2 2 ( 2 5 \%}$ & $392(95 \%)$ & $\mathbf{1 1 ( 3 \% )}$ \\
$110-125$ & $61(57 \%)$ & $\mathbf{1 2}(\mathbf{2 0 \%}$ & $411(99 \%)$ & $\mathbf{2 8}(\mathbf{7 \%})$ \\
$125-140$ & $30(28 \%)$ & $5(17 \%)$ & $411(99 \%)$ & $52(13 \%)$ \\
$140-155$ & $8(7 \%)$ & $1(12 \%)$ & $408(99 \%)$ & $60(15 \%)$ \\
$155-170$ & $5(5 \%)$ & $0(0 \%)$ & $387(93 \%)$ & $110(28 \%)$ \\
$170-185$ & $0(0 \%)$ & $0(0 \%)$ & $344(83 \%)$ & $92(27 \%)$ \\
$185-200$ & $0(0 \%)$ & $0(0 \%)$ & $226(55 \%)$ & $21(9 \%)$ \\
$200-215$ & $0(0 \%)$ & $0(0 \%)$ & $44(11 \%)$ & $1(2 \%)$ \\
$215-230$ & $0(0 \%)$ & $0(0 \%)$ & $1(0.2 \%)$ & $0(0 \%)$ \\
\hline
\end{tabular}

An important fact, as could be seen from the Table is that only $8.4 \%$ of non-athletes achieve a HR > $140 \mathrm{bpm}$ during stress test, compared to almost all the athletes (99\%). Only one TWA cluster was detected in non-athletes with a HR > $140 \mathrm{bmp}$, while most of the TWA clusters in athletes occur exactly in this HR range.

We highlighted in red three rows in Table 2, with a HR range 80-125, where TWA occurs in both groups. Limiting the analysis to this HR range, we found a significantly lower TWA prevalence in athletes compered to nonathletes: $50(12.1 \%)$ vs $46(42.9 \%), \mathrm{p}<0.001$.

Comparing TWA clusters' characteristics in the HR range of interest (80-125 bpm), we observed lower max and mean TWA amplitudes in athletes compared to nonathletes - Table 3.

Table 3. TWA characteristics in athletes and non-athletes considering TWA clusters in the HR range $80-125 \mathrm{bpm}$. (Median values, [25th -75 th] percentiles). ( $<<0.01$; $\left.\mathrm{p}<0.05^{* *}\right)$.

\begin{tabular}{lccc}
\cline { 2 - 4 } & $\begin{array}{c}\text { Athletes } \\
(\mathrm{n}=50)\end{array}$ & $\begin{array}{c}\text { Non- } \\
\text { athletes } \\
(\mathrm{n}=46)\end{array}$ & $\mathrm{p}$ \\
\hline TWA mean ampl. & $0.32 \mu \mathrm{V}$ & $0.37 \mu \mathrm{V}$ & - \\
& {$[0.10-0.90]$} & {$[0.16-0.67]$} & \\
TWA max ampl. & $0.51 \mu \mathrm{V}$ & $0.52 \mu \mathrm{V}$ & - \\
& {$[0.21-2.19]$} & {$[.32-1.04]$} & \\
Mean HR & $126 \mathrm{bpm}$ & $100 \mathrm{bpm}$ & $*$ \\
& {$[110-139]$} & {$[91-105]$} & \\
HR in 1 ${ }^{\text {st }}$ packet & $111 \mathrm{bpm}$ & $101 \mathrm{bpm}$ & $* *$ \\
& {$[96-120]$} & {$[93-110]$} & \\
HR in 2 ${ }^{\text {nd }}$ packet & $146 \mathrm{bpm}$ & $91 \mathrm{bpm}$ & $*$ \\
& {$[121-157]$} & {$[79-102]$} & \\
\hline
\end{tabular}

\section{Discussion}

In the present study, we analysed the prevalence of TWA during CPT in athletes and compared it with a group with high expected TWA prevalence - patients with multiple risk factors, with suspected or proven coronary artery disease, performing diagnostic stress electrocardiographic test. The detection of TWA clusters showed an unexpected high overall prevalence of TWA, with worse TWA parameters, in athletes as compared to non-athletes. This was a surprising finding, since most of the participants in the non-athletes' group had a diagnosed heart disease or other conditions, implying high cardiovascular risk.

These results could be explained by different test conditions: due to fatigue and frequent concomitant use of beta-blockers, the duration of the test in non-athletes is interrupted long before reaching the maximal heart rate/load (test duration in athletes is almost twice as longer as that in non-athletes). This allows more time for TWA clusters to appear in the first group. On the other hand, TWA occurrence depends on HR - as higher is the HR, as greater is the possibility for TWA. Due to premature interruption of the test (caused by fatigue) and to slower load-dependent rise of HR in non-athletes, athletes achieved significantly higher HR during stress test compared to non-athletes.

We performed further analysis, comparing the occurrence of TWA clusters only in the HR range (80-125 bpm) that had been achieved during exercise both in the athletes' and in the non-athletes' groups.

This HR-matched comparison changed radically the results, rendering non-athletes with a higher rate of occurrence of TWA clusters, and worse TWA parameters, 
than athletes.

Several aspects of our research deserve special attention: first, we observed a high prevalence of TWA clusters in athletes during CPT; second, the study raises concerns about the validity of the results when TWA occurrence is compared between groups of subjects in different loading conditions; and finally, considering that nearly $80 \%$ of the elite athletes in our group had at least one TWA cluster during CPT, maybe it is time to reconsider the prognostic significance of this parameter or to refine the detection algorhythm.

\section{Conclusion}

TWA is a HR-dependent parameter and athletes achieving high HR during CPT have an increased prevalence of TWA. Therefore, the prognostic significance of exercise-induced TWA in athletes could not be the same as that of TWA in sedentary high-risk non-athletes, where TWA appears in a significantly lower HR range.

\section{Acknowledgements}

This study was supported by a joint project between the Italian Research Council (Institute of Neuroscience IN$\mathrm{CNR}$ ) and the Bulgarian Academy of Sciences (Institute of Biophysics and Biomedical Engineering).

\section{References}

[1] Verrier R., Klingenheben T., Malik M., et al. Microvolt TWave Alternans. Physiological Basis, Methods of Measurement, and Clinical Utility-Consensus Guideline by International Society for Holter and Noninvasive Electrocardiology. J Am Coll Cardiol. 2011;58(13):13091324

[2] Slawnych MP, Nieminen T, Kähönen M, et al; REFINE (Risk Estimation Following Infarction Noninvasive Evaluation); FINCAVAS (Finnish Cardiovascular Study) Investigators. Post-exercise assessment of cardiac repolarization alternans in patients with coronary artery disease using modified moving average the method. J Am Coll Cardiol. 2009; 53(13):1130-7

[3] Minkkinen M., Kähönen M., Viik J., et al. Enhanced Predictive Power of Quantitative TWA during Routine Exercise Testing in the Finnish Cardiovascular Study. J Cardiovasc Electrophysiol 2009; 20 (4):408-415.

[4] Verrier R., Klingenheben T., Malik M., et al. Microvolt TWave Alternans Testing Has a Role in Arrhythmia Risk Stratification. J Am Coll Cardiol. 2012; 59(17):1572-1573
[5] Simov D. Electrocardiographic changes in certain cardiovascular physiological and pathological settings. Impact on coronary artery bypass grafting. Int $\mathrm{J}$ Bioautomation. 2016;20(1):43-68.

[6] Simov D, Christov I, Bortoan G, et al. Changes in the electrocardiogram induced by coronary artery bypass grafting. Comp. Card. 2015; 42:1129-1132.

[7] Simova I, Bortoan G, Kambova L, et al. Episodes of T-wave and QRS complex alternans in haemodialysis patients. EC Cardiol. 2015;2(1):60-67.

[8] Christov I, Bortolan G, Simova I, Katova T. Influence of diabetes mellitus on $\mathrm{T}$ wave and QRS complex alternans during stress ECG testing. Comp. Cardiol. 2011; 38:49-52.

[9] Christov I, Bortolan G, Simova I, Katova T. T-wave and QRS complex alternans during standard diagnostic stress ECG test. Comp. Cardiol. 2010; 37:1039-1042.

[10] Bortolan G, Christov II, Batchvarov VN, Behr ER. QRS\&T wave alternans and beat-to-beat ventricular repolarization variability assessed from 12-lead Holters in patients with suspected Brugada syndrome. Comp. Cardiol. 2009; 36:305308

[11] Pelliccia A., Adami P., Quattrini F. et al. Are Olympic athletes free from cardiovascular diseases? Systematic investigation in 2352 participants from Athens 2004 to Sochi 2014.Br J Sports Med 2017; 51:238-243

[12] Inama G, Pedrinazzi C, Durin O, et al. Microvolt T-wave alternans for risk stratification in athletes with ventricular arrhythmias: Correlation with programmed ventricular stimulation. Ann. Noninv. Electrocard. 2008;13(1):14-21.

[13] Levkov Ch, Mihov G, Ivanov R, et al. Removal of powerline interference from the ECG: A review of the subtraction procedure. Biomed Eng Online. 2005; 4(50)

[14] Christov II, Daskalov IK. Filtering of electromyogram artifacts from the electrocardiogram, Med. Eng. \& Phys. 1999; 21(10):731-736.

[15] Daskalov IK, Dotsinsky IA, Christov II. Developments in ECG acquisition, preprocessing, parameter measurement and recording, IEEE Eng. in Med. \& Biol. 1998; 17(2):5058 .

[16] Bortolan G, Christov II. Principal component analysis for the detection and assessment of T-wave alternans. Comp. Card. 2008; 35:521-524

[17] Bortolan G, Christov II. T-wave alternans detection by a combined method of principal component analysis and Twave amplitude. Physiol. Meas. 2012; 33: 333-343

Address for correspondence.

Associate professor Iana Simova, MD, PhD; Acibadem City

Clinic Cardiovascular Center - University Hospital, 127

Okolovrasten Put Str, Sofia 1407, Bulgaria

ianasimova@gmail.com 